\title{
Comparative Analysis of the Therapeutic Effects of Amniotic Membrane and Umbilical Cord Derived Mesenchymal Stem Cells for the Treatment of Type 2 Diabetes
}

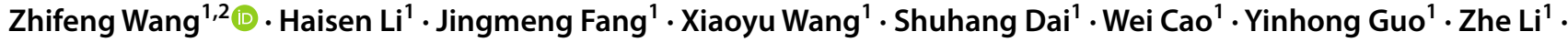 \\ Hao Zhu' ${ }^{1}$
}

Accepted: 16 December 2021 / Published online: 11 January 2022

๑) The Author(s), under exclusive licence to Springer Science+Business Media, LLC, part of Springer Nature 2022

\begin{abstract}
Type 2 diabetes mellitus (T2DM), one of the most common carbohydrate metabolism disorders, is characterized by chronic hyperglycemia and insulin resistance (IR), and has become an urgent global health challenge. Mesenchymal stem cells (MSCs) originating from perinatal tissues such as umbilical cord (UC) and amniotic membrane (AM) serve as ideal candidates for the treatment of T2DM due to their great advantages in terms of abundant source, proliferation capacity, immunomodulation and plasticity for insulin-producing cell differentiation. However, the optimally perinatal MSC source to treat T2DM remains elusive. This study aims to compare the therapeutic efficacy of MSCs derived from AM and UC (AMMSCs and UCMSCs) of the same donor in the alleviation of T2DM symptoms and explore the underlying mechanisms. Our results showed that AMMSCs and UCMSCs displayed indistinguishable immunophenotype and multi-lineage differentiation potential, but UCMSCs had a much higher expansion capacity than AMMSCs. Moreover, we uncovered that single-dose intravenous injection of either AMMSCs or UCMSCs could comparably reduce hyperglycemia and improve IR in T2DM $\mathrm{db} / \mathrm{db}$ mice. Mechanistic investigations revealed that either AMMSC or UCMSC infusion could greatly improve glycolipid metabolism in the liver of $\mathrm{db} / \mathrm{db}$ mice, which was evidenced by decreased liver to body weight ratio, reduced lipid accumulation, upregulated glycogen synthesis, and increased Akt phosphorylation. Taken together, these data indicate that the same donor-derived AMMSCs and UCMSCs possessed comparable effects and shared a similar hepatoprotective mechanism on the alleviation of T2DM symptoms.
\end{abstract}

Zhifeng Wang, Haisen Li and Jingmeng Fang contributed equally to this work.

Zhifeng Wang

wangzhifeng68@hotmail.com

$\triangle$ Hao Zhu

zhuhao@sinoneural.com

1 Sinoneural Cell Engineering Group Holdings Co., Ltd, Shanghai 201100, China

2 Translational Medical Center for Stem Cell Therapy and Institute for Regenerative Medicine, Shanghai East Hospital, Shanghai Key Laboratory of Signaling and Disease Research, Frontier Science Center for Stem Cell Research, School of Life Sciences and Technology, Tongji University, Shanghai 200092, China 


\section{Graphical Abstract}

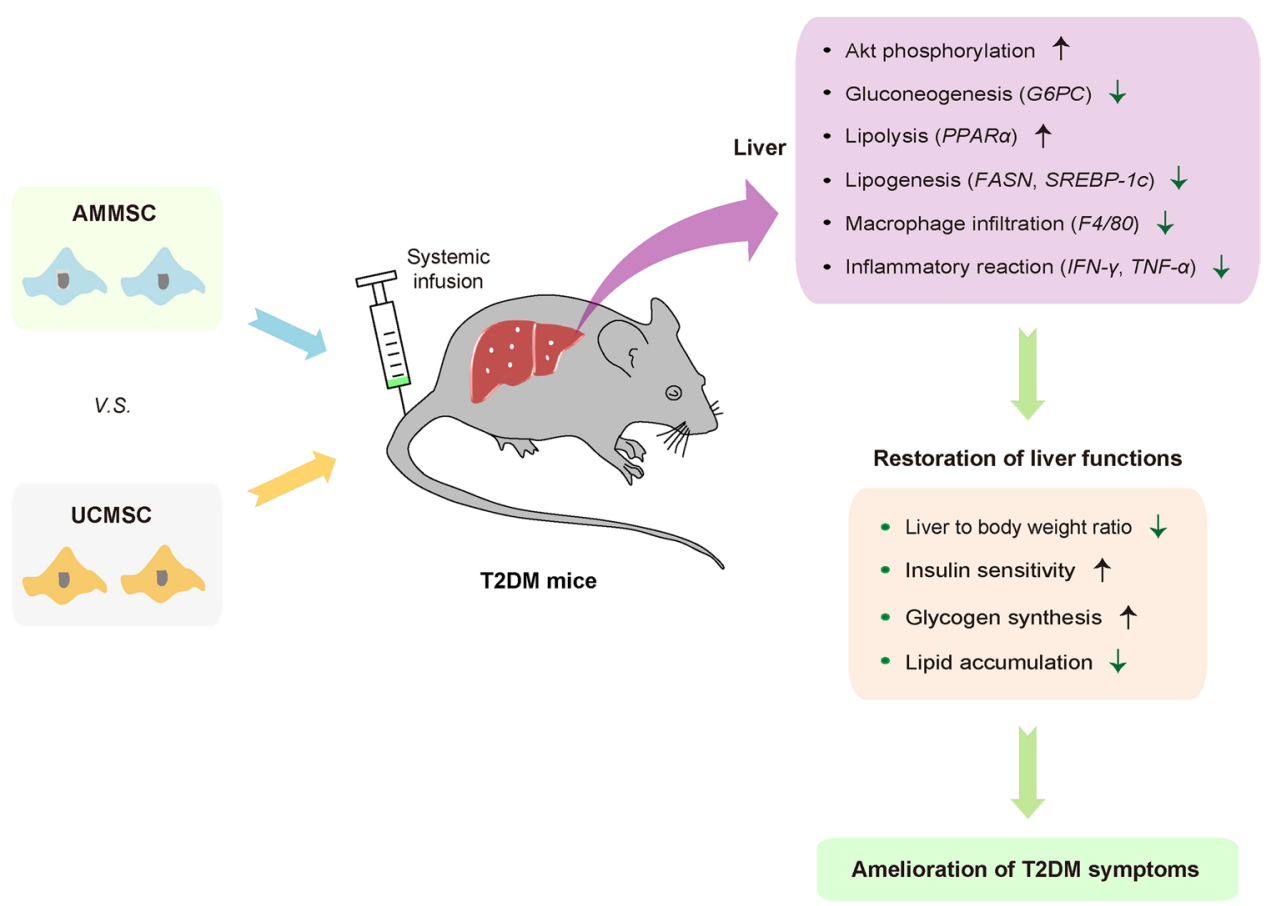

Keywords Mesenchymal stem cells $\cdot$ Umbilical cord $\cdot$ Amniotic membrane $\cdot$ Type 2 diabetes $\cdot$ Hyperglycemia $\cdot$ Liver

\section{Introduction}

Global estimates indicate that over 462 million people are currently suffering from diabetes mellitus, and this number expectedly rises to around 700 million by 2045 [1, 2]. T2DM, the most common form of diabetes, is pathologically caused by IR and pancreatic $\beta$-cell destructions, which eventually lead to the uncontrolled hyperglycemia in T2DM [2, 3]. IR is well known as the foremost pathogenesis of T2DM, and characterized by the impaired ability of peripheral tissues to response to insulin [2, 4], thereby causing the failure of glucose uptake within insulin-responsive tissues such as white adipose tissue, muscle, and liver. Among these organs, the liver functions as a vital player in the regulation of glycolipid homeostasis by directing nutrients to storage as triglycerides and glycogen $[5,6]$. Besides, more than $90 \%$ of T2DM patients with obesity are described to have metabolic dysfunction-associated fatty liver disease [7], indicating a close association of T2DM with hepatic dysfunctions. Hepatic IR contributes greatly to the enhanced gluoconeogenesis and fatty acid synthesis in the hepatocytes of T2DM individuals [8], which accordingly conduces to elevated hepatic glycogen decomposition and lipid accumulation. Nowadays, many anti-diabetic drugs such as metformin, liraglutide and ipragliflozin, have been developed to alleviate T2DM through reversing peripheral IR and partially restoring $\beta$-cell mass [9]. However, these current first-line drugs are unable to cure T2DM, and their lifelong usage may evoke a high risk of severe side effects such as gastrointestinal reaction and life-threating hypoglycemia $[2,10]$. Thus, it is extremely urgent to develop novel therapeutic approaches to maintain long-term normoglycemia and even cure T2DM.

Due to their self-renewal, differentiation, immunosuppression and paracrine characteristics, MSCs have emerged as a very promising therapeutic modality to treat various human diseases including metabolic, cardiovascular, neurodegenerative, and autoimmune disorders [2, 11-16]. Intriguingly, human MSCs have been demonstrated to obviously decrease acute respiratory distress and mortality in patients with COVID-19 through improving pulmonary functions and reducing inflammatory biomarker levels [17, 18]. MSCs, existing in connective tissues of most organs, have been successfully obtained from adult tissues such as bone marrow (BM), lung and adipose, and perinatal tissues including AM, UC, and placenta [19-21]. In comparison with adult tissues-isolated MSCs, perinatal MSCs exhibit numbers of advantages, including, but not limited to, lower immunogenicity, higher proliferation capacity, greater differentiation potential, easily accessible, and abundant source 
[20-25]. Human UCMSCs have been shown to exert better therapeutic effects than BM-derived MSCs in patients with type 1 DM (T1DM) $[1,26]$. Moreover, human UCMSC administration has been demonstrated to efficiently ameliorate systemic IR and hyperglycemia in rodent T2DM models by enhancing insulin sensitivity, suppressing inflammation, and improving glycolipid metabolism dysfunction in the liver [27-30]. Human AMMSCs are found to accelerate the skin wound healing in a T1DM mouse model [31], and the transplantation of human AMMSCs-differentiated insulinproducing cells or islet-like cell aggregates could normalize hyperglycemia in T1DM mice [32,33]. And the application of human AM has been described to facilitate the ulcer healing in T2DM patients with chronic ulcers [34]. However, it remains unclear whether the AMMSC administration has therapeutic benefits for the treatment of T2DM.

In the present study, to determine the potential benefits of AMMSCs and optimally perinatal source of MSCs in T2DM therapy, we compared the therapeutic effects of human AMMSCs and UCMSCs derived from the same donor in leptin receptor-deficient $\mathrm{db} / \mathrm{db}$ mice spontaneously developing many severe humanlike T2DM symptoms. Our results showed that either AMMSC or UCMSC administration could comparably alleviate T2DM symptoms through enhancing systemic insulin sensitivity and reducing hyperglycemia. Mechanistically, the therapeutic benefits of perinatal MSCs could be attributed to the improvement of aberrant glycolipid metabolism, enhanced Akt phosphorylation, and suppressed inflammation in the liver of $\mathrm{db} / \mathrm{db}$ mice. Overall, our data not only manifested the similar therapeutic effectiveness and mechanisms of the same donor-derived AMMSCs and UCMSCs in the treatment of T2DM mice, but also provided very useful hints for the clinical application of human perinatal MSCs in treating T2DM.

\section{Materials and Methods}

\section{Human MSC Isolation and Characterization}

Human AM and UC tissues were acquired from healthy post-natal female with written informed consent. Human AMMSCs and UCMSCs were isolated and cultured according to previously described methods [35]. Primary AMMSCs and UCMSCs were expanded in MSC NutriStem ${ }^{\circledR}$ XF Medium (Biological Industries) supplemented with 5\% Knockout ${ }^{\mathrm{TM}}$ Serum Replacement (Thermo Fisher Scientific).

AMMSCs and UCMSCs at the fifth passage were utilized for the assessments of MSC surface markers and trilineage differentiation capacity. For the immunophenotypes of MSCs, the expressions of positive cell-surface markers (CD44, CD73, CD90, CD105 and CD166) and negative markers (CD14, CD19, CD34, CD45 and HLA-DR) were determined with the flow cytometer (BD FACS Canto II flow cytometer). All above antibodies were conjugated with phycoerythrin (PE) and purchased from BioLegend. Tri-lineage differentiation capacity of AMMSCs and UCMSCs was assessed with specific differentiation kits (Thermo Fisher Scientific), based on the manufacturers' instructions. The osteocytes, adipocytes, and chondrocytes were identified by Alizarin Red S staining, Oil Red O staining, and Alcian Blue staining, respectively.

\section{Growth Curve Analysis of MSCs}

UCMSCs and AMMSCs were plated into 6-well culture plates at the density of $6 \times 10^{4}$ cells per well. Cell numbers in three individual wells were counted daily for continuous 5 days by the AO/PI method. Cell growth curves were then plotted.

\section{Gene Expression Profiles of MSCs Stimulated with/ without Interferon-gamma (IFN- $\gamma$ )}

To compare the responses of AMMSCs and UCMSCs to IFN- $\gamma$, the mRNA expression levels of immunoregulatory and growth factor genes were determined using quantitative real-time reverse transcription PCR (qRT-PCR) method. Briefly, MSCs at the third passage were plated into 6-well culture plates $\left(3 \times 10^{5}\right.$ cells per well). After overnight growth, $10 \mathrm{ng} / \mathrm{ml}$ IFN- $\gamma$ (Sigma-Aldrich) was added to each well. 24 hours later, IFN- $\gamma$-stimulated MSCs were washed with ice-cold PBS for three times, and harvested through adding $1 \mathrm{~mL}$ TRIzol reagent (Invitrogen) into each well. The qRT-PCR targets for growth factors include hepatocyte growth factor $(H G F)$, vascular endothelial growth factor (VEGF), insulin-like growth factor 1 (IGF1) and fibroblast growth factor 2 (FGF2), while immunoregulatory genes involve interleukin-6 (IL-6), IL-11, transforming growth factor- $\beta$ (TGF- $\beta)$, and indoleamine 2,3-dioxygenase (IDO1).

\section{Animals and MSC Administration}

All the experimental procedures on mice had been reviewed and approved by the Laboratory Animal Care and Use Committee of Tongji University. Mouse care and experiments were undertaken by conforming to the National Institutes of Health Guide for the Care and Use of Laboratory Animals. Male 6-week-old C57BLKS/JNju (normal) and BKS-Lepr ${ }^{\text {em2Cd479 }} / \mathrm{Nju}(\mathrm{db} / \mathrm{db})$ mice were obtained from GemPharmatech $^{\mathrm{TM}}$ (Nanjing, China). All mice were housed in the constant temperature $\left(20 \pm 2^{\circ} \mathrm{C}\right)$ and humidity $(50 \%)$ room with a 12-hour artificial light-dark cycle. All mice were fed with a standard diet, and allowed to freely acquire food and drinking water. 
AMMSCs or UCMSCs at the fifth passage were injected into 7 -week-old $\mathrm{db} / \mathrm{db}$ mice via the tail vein at a single dose of $1.5 \times 10^{6}$ cells $/ 0.2 \mathrm{ml}$ saline. Following this way, $0.2 \mathrm{ml}$ saline was administrated intravenously into $\mathrm{db} / \mathrm{db}$ and normal mice (control groups). Subsequently, the body weights and random blood glucose concentrations of MSCs- or Saline-infused mice were monitored at indicated time points.

\section{Analyses of Metabolic Parameters}

Intraperitoneal glucose tolerance test (IPGTT) and intraperitoneal insulin tolerance test (IPITT) were undertaken in MSCs- and Saline-injected mice after two weeks of treatment. For the IPGTT, mice were starved for overnight, and their tails were bled to determine the initial blood glucose concentration with a Accu-Chek Mobile glucometer (Roche). Glucose ( $2 \mathrm{~g} / \mathrm{kg}$ bodyweight) was administrated into the mice via intraperitoneal injection, and blood glucose level was then monitored. For the IPITT, mice were firstly conducted with 6-hour starvation, then injected with 0.5-1.5 unit $/ \mathrm{kg}$ of human insulin (Novolin 30R, Novo Nordisk), and the blood glucose concentration was measured.

\section{Mouse Tissue Collection}

For collecting mouse tissues, the mice were anesthetized after five weeks of MSC administration, and their epididymal white adipose tissue (EWAT) and liver were isolated and weighed. A portion of mouse livers was promptly cut into small pieces and snap-frozen in liquid nitrogen for below gene expression analyses, while the other part was fixed in formalin for below histological experiments. For western blotting assay, mice were firstly starved for 6 hours, and then administrated with human insulin ( 1 unit/kg bodyweight, Novolin 30R, Novo Nordisk). One hour later, mouse livers were collected and kept in the liquid nitrogen by snapfreezing for below protein purification.

\section{qRT-PCR Assay}

Total RNAs were purified from human MSCs or mouse livers with the TRIzol reagent, and subsequently reverse transcribed using the RevertAid First Strand cDNA Synthesis Kit (Thermo Fisher Scientific) based on its manufacturer's instruction. qRT-PCR primers were designed via utilizing the NCBI's Primer-BLAST tool, and then synthesized by Sangon Biotech Co., Ltd (Shanghai). The GoTaq qPCR Master Mix Kit (Promega) was employed for the PCR reactions, and the relative expression values of target genes were calculated by following the $2^{-\Delta \Delta \mathrm{Ct}}$ method. The sequences of qRT-PCR primers in this study are listed in Supplementary Table 1.

\section{Hematoxylin and Eosin Stain (H\&E) and Periodic-Acid Schiff (PAS) Staining}

Mouse livers were firstly processed in the formalin (Solarbio) and then embedded in the paraffin tissue blocks. $4 \mu \mathrm{m}$-thick sections were cut using a microtome, and transferred onto Bond-Rite ${ }^{\mathrm{TM}}$ slides. After being heated at $60^{\circ} \mathrm{C}$ for 1 hour, the sections were deparaffinized in fresh xylene (three changes), and then rehydrated with descending grades of alcohol. Next, the sections were performed with either PAS (Solarbio) or H\&E (Servicebio) staining, and comparatively viewed under the Olympus microscope equipped with an Olympus digital camera. Three areas having the same size were picked casually from each liver section, and their staining density was counted with the Image J software. The average value of lipid droplets within H\&E staining or glycogenpositive area in PAS staining was utilized to respectively determine total lipid or glycogen content of mouse liver.

\section{Western Blotting}

Total proteins were extracted from mouse livers by using PMSF-contained RIPA lysis buffer (Beyotime), and their concentration was measured employing the BCA Protein kit (Beyotime). Total proteins were resolved by $10 \%$ SDSPAGE, and then transferred onto the PVDF membrane. The PVDF membranes were sequentially probed with primary and secondary antibodies. Primary antibodies were purchased from Cell Signaling Technology and listed as follows: phospho-Akt (\#9271s, 1:1000), Akt (\#2920s, 1:1000), and GAPDH (1:5000). After being probed with the secondary antibody (Abcam, 1:5000) for 1 hour, the membranes were washed three times in TBST, and lastly detected with the Enhanced Chemiluminescent Detection Kit (Thermo Fisher Scientific).

\section{Statistical Analyses}

Quantitative results were displayed as mean \pm standard error of mean (SEM) from at least three independent samples. The significant differences of group differences were determined by the unpaired t-test between two groups, or one-way ANOVA followed by LSD post hoc test for multiple groups. P-value $(<0.05)$ was thought to be statistically significant.

\section{Results}

\section{Characterization of the Same Donor-derived AMMSCs and UCMSCs}

To determine whether these culture-expanded cells possess typical MSC characteristics, we evaluated their morphology, 
surface antigens and multiple differentiation capacities. Both UCMSCs and AMMSCs were attached to cell culture plates, and exhibited a spindle-shaped and fibroblast-like morphology (Fig. 1A). Consistent with typical MSC immunophenotype, more than $99.5 \%$ of UCMSCs and AMMSCs were positive for CD44, CD73, CD90, CD105 and CD166, while less than $0.25 \%$ were positive for CD14, CD19, CD34, CD45 and HLA-DR (Fig. 1B). Meanwhile, both AMMSCs and UCMSCs exhibited indistinguishable capacity to differentiate into adipocytes, chondrocytes and osteocytes in vitro (Fig. 1C). These characteristics of AMMSCs and UCMSCs were completely in conformity with the International Society of Cell Therapy (ISCT) criteria [2], demonstrating their MSC properties. Collectively, these results indicated that the same donor-derived AMMSCs and UCMSCs have indistinguishable phenotypes and tri-lineage differentiation capacity.

\section{Proliferation and Gene Expression Profiles of AMMSCs and UCMSCs}

Next, we tried to compare the proliferative and selected gene expression patterns between AMMSCs and UCMSCs. The growth curve showed that UCMSCs proliferated faster than the same donor-derived AMMSCs (Fig. 2A). Additionally, we assessed the expression patterns of selected growth factors and anti-inflammatory genes within AMMSCs and UCMSCs in the absence or presence of pro-inflammatory cytokine IFN- $\gamma$, which has been
A
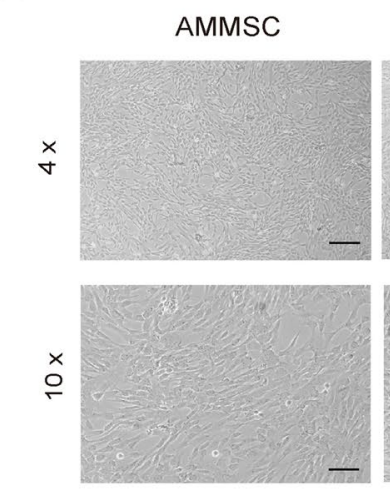

C
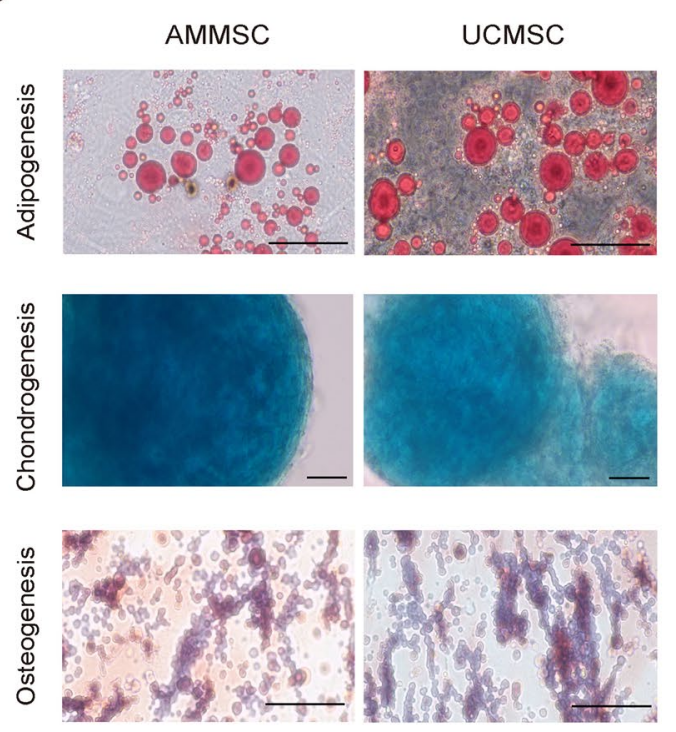

UCMSC
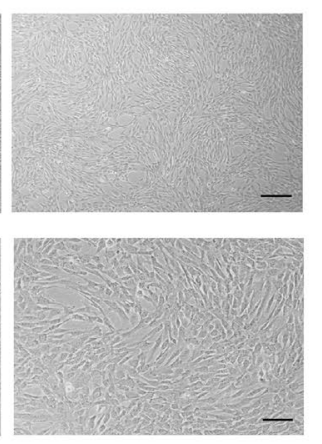

B

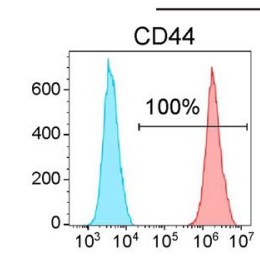

AMMSC
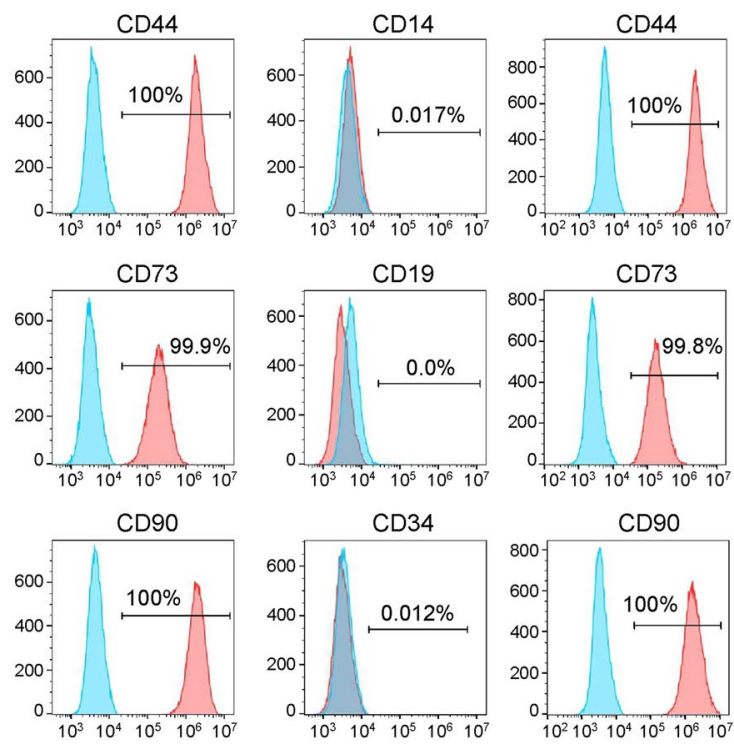
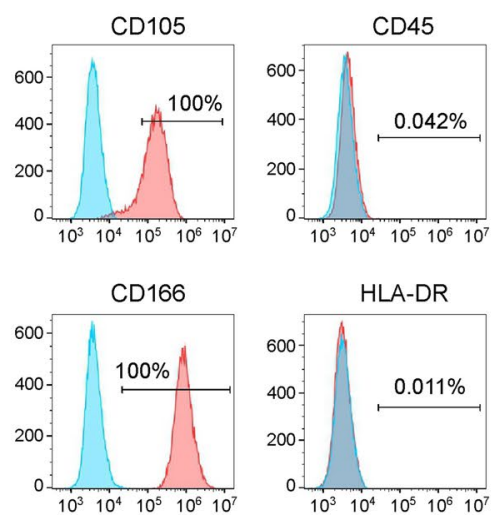
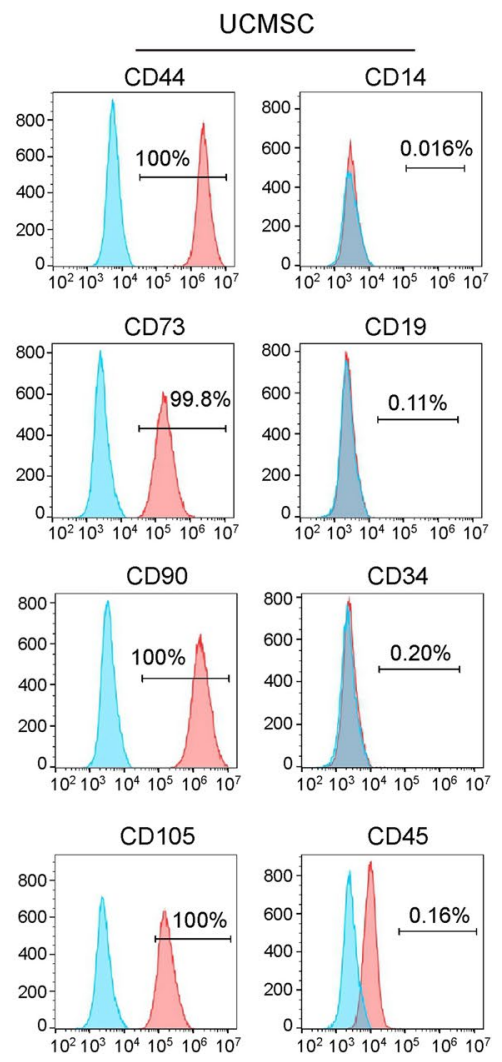

UCMSC
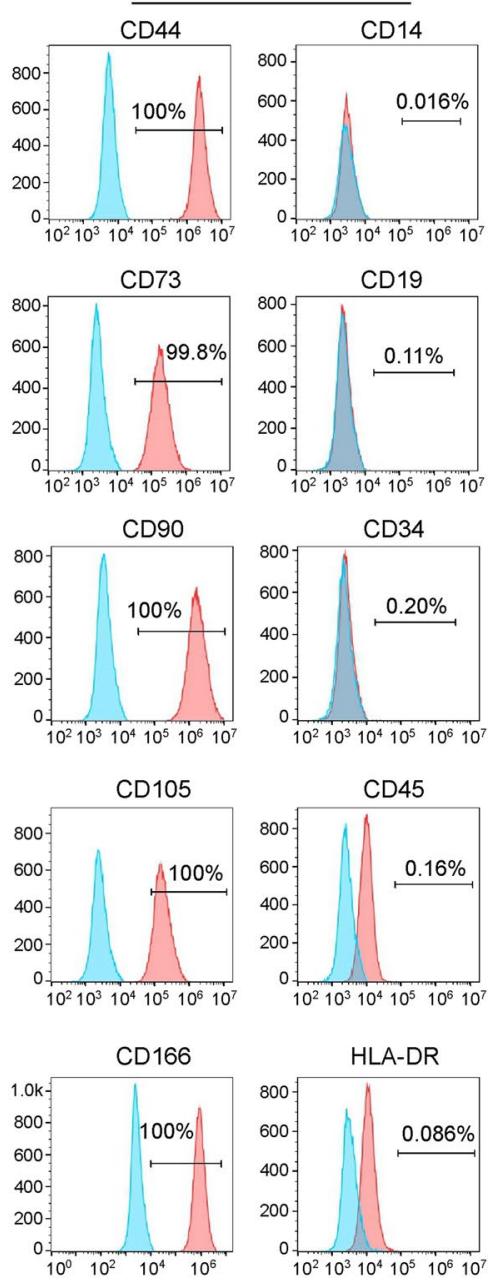

Fig. 1 Biological characterization of AMMSCs and UCMSCs originating from the same donor. (A) Representative pictures of the morphology of AMMSCs and UCMSCs. The pictures were taken with a light microscope at $4 \times$ (top) and $10 \times$ (bottom) magnification. Scale bar $=100 \mu \mathrm{m}$. (B) Representative histograms of immunophenotypic characterization of AMMSCs and UCMSCs by flow cytometry analysis. Blue areas indicate isotype control staining, and red areas represent specific marker expression. (C) Representative images of adipocytes (top), chondrocytes (middle) and osteocytes (bottom) differentiated from AMMSCs or UCMSCs. The images were captured at $40 \times$ (top), $20 \times$ (middle) and $40 \times$ (bottom) magnification, respectively. Scale bar $=50 \mu \mathrm{m}$ 
Fig. 2 The growth curves and gene expression patterns of AMMSCs and UCMSCs.

(A) Growth curve analyses of AMMSCs and UCMSCs. (B-I) mRNA expression levels of $H G F(\mathbf{B}), I G F 1$ (C), FGF2 (D), $\operatorname{VEGF}(\mathbf{E}), I L-6(\mathbf{F}), I L-11$ (G), IDOI (H) and TGF- $\beta$ (I) in AMMSCs and UCMSCs with or without IFN- $\gamma$ stimulation. The results are shown as mean $\pm \operatorname{SEM}(\mathrm{n}=3) . * P<0.05, * * P$ $<0.01, * * * P<0.001$
A

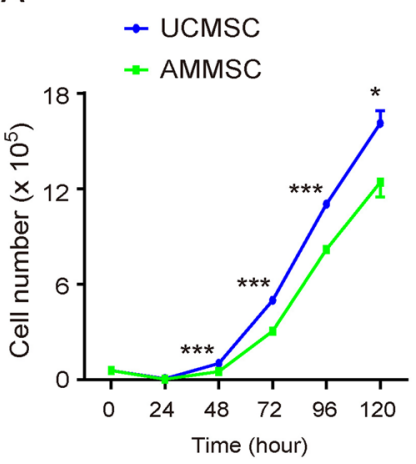

D

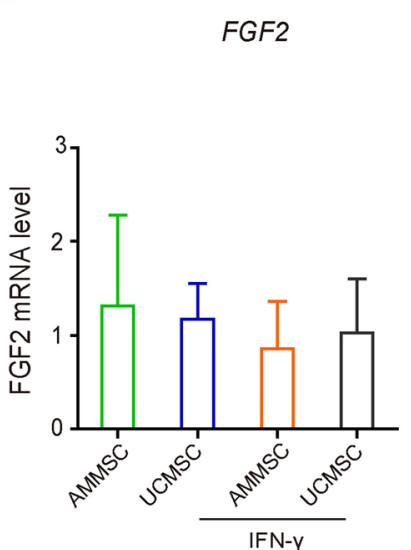

G

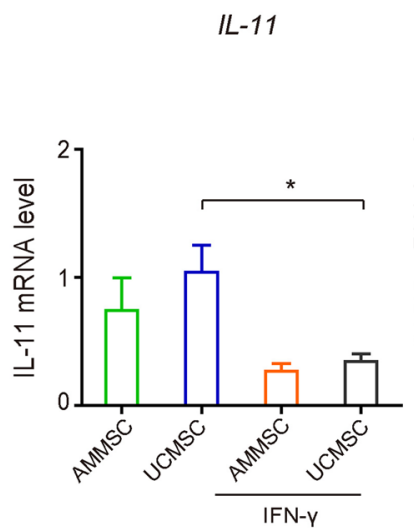

B

HGF

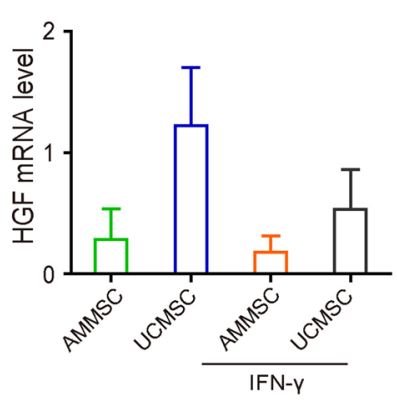

E

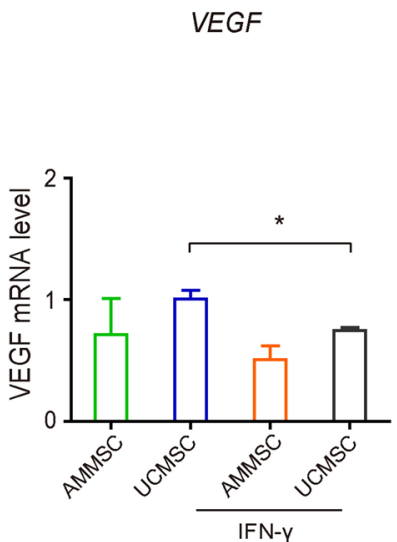

$\mathrm{H}$

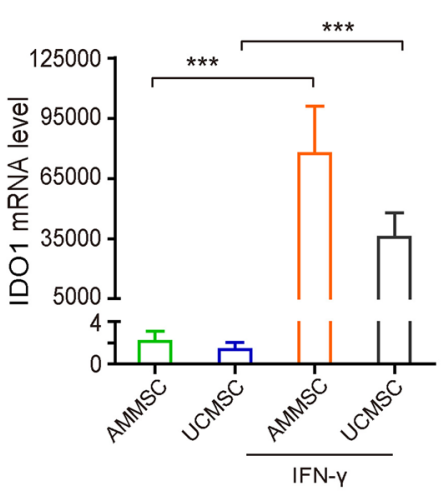

C

IGF1

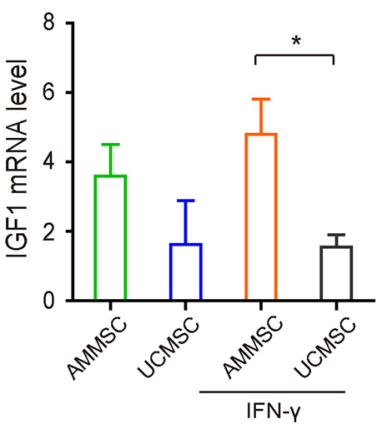

$\mathrm{F}$

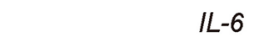

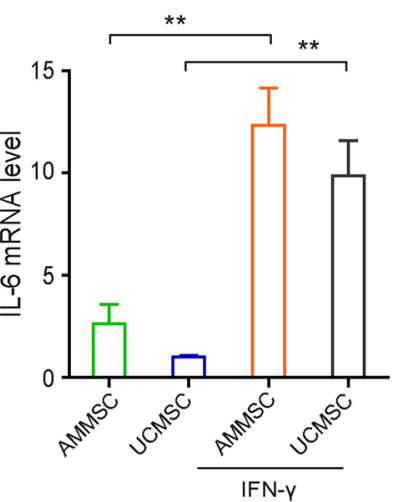

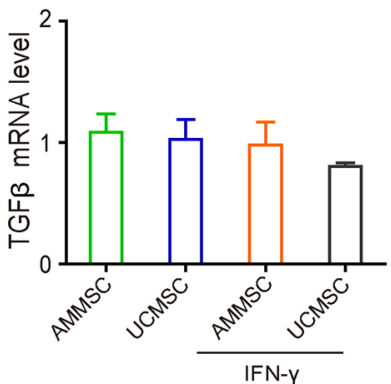

extensively utilized as a pre-licensing agent to improve MSC immunosuppressive activity [36-38]. Under basal condition, the mRNA expression levels of growth factors and anti-inflammatory genes were similar between AMMSCs and UCMSCs except $H G F$ with a higher expression level in UCMSCs than AMMSCs (Fig. 2B-I). After 24-hour IFN- $\gamma$ stimulation, the mRNA expression levels of $I L-6$ and $I D O I$ were dramatically increased, but $I L-1 I$ was reduced in both AMMSCs and UCMSCs (Fig. 2FH). AMMSCs displayed much higher IGFI level than
UCMSCs in response to IFN- $\gamma$ stimulation (Fig. 2C). IFN- $\gamma$ treatment significantly decreased $V E G F$ expression level in UCMSCs rather than in AMMSCs (Fig. 2E). IFN- $\gamma$ stimulation could not significantly change the mRNA levels of $H G F, I G F 1, F G F 2$ and $T G F-\beta$ in either AMMSCs or UCMSCs (Fig. 2B-D and I). Therefore, these data suggested that UCMSCs have faster proliferative rate than AMMSCs, but they possessed similar gene expression patterns of growth and anti-inflammatory factors except $I G F 1$ under IFN- $\gamma$ stimulation. 


\section{MSC Infusion Alleviated Hyperglycemia and IR in $\mathbf{d b} / \mathbf{d b}$ Mice}

To compare therapeutic effects of AMMSC and UCMSC infusion on T2DM, 7-week-old male $\mathrm{db} / \mathrm{db}$ mice were intravenously infused with a single dose of either AMMSCs or UCMSCs. The $\mathrm{db} / \mathrm{db}$ mice receiving UCMSC infusion showed markedly reduced blood glucose level at 7 days post-injection when compared to the saline control, and this hypoglycemic activity could persist for 5 weeks (Fig. 3A).
Likewise, AMMSC injection led to a similar hypoglycemic effect in $\mathrm{db} / \mathrm{db}$ mice (Fig. 3A). There was no difference in the body weight between saline- and MSCs-injected db/ $\mathrm{db}$ mice (Fig. 3B). Supporting the improved hyperglycemia, either AMMSC or UCMSC administration had a great benefit on insulin sensitivity and glucose tolerance in $\mathrm{db} / \mathrm{db}$ mice (Fig. 3C-F). Taken together, these findings suggested that the same donor-derived AMMSCs and UCMSCs could exert comparably alleviative effects on hyperglycemia and IR in $\mathrm{db} / \mathrm{db}$ mice.
Fig. 3 Metabolic phenotypes of $\mathrm{db} / \mathrm{db}$ mice after AMMSC or UCMSC administration. Random blood glucose concentrations (A) and body weights (B) of different group mice were determined at indicated time points. Changes of blood glucose concentrations $(\mathbf{C})$ and area under the curve (D) from the IPITT in different group mice at two weeks after MSC or saline infusion. Changes of blood glucose levels $(\mathbf{E})$ and area under the curve $(\mathbf{F})$ from the IPGTT in mice of different groups at two weeks after MSC or saline infusion. Data are displayed as mean \pm SEM. Normal group: $\mathrm{n}=6$ mice; Saline group: $\mathrm{n}=7$ mice; UCMSCs group: $\mathrm{n}=7$ mice; AMMSCs group: $\mathrm{n}=8$ mice. AUC: area under the curve. ${ }^{*} P$ or ${ }^{\#} P<0.05,{ }^{* *} P$ or ${ }^{\# \#} P<0.01$, $* * * P<0.001$. In $(\mathbf{A})$ and $(\mathbf{C})$, *: UCMSCs group vs. saline group; \#: AMMSCs group vs. saline group
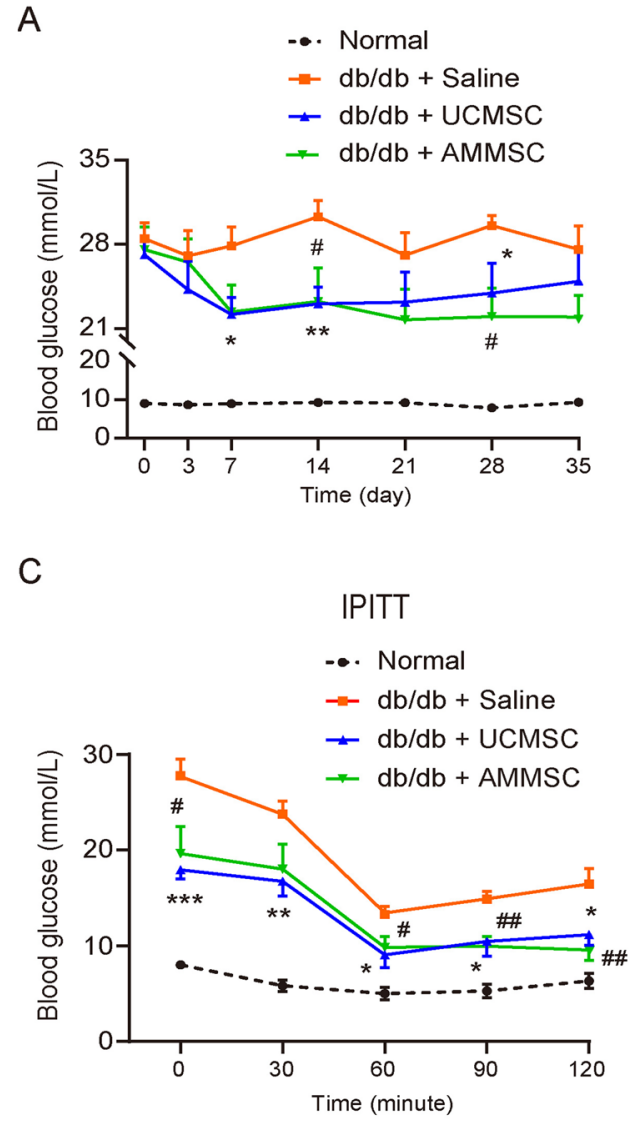

E

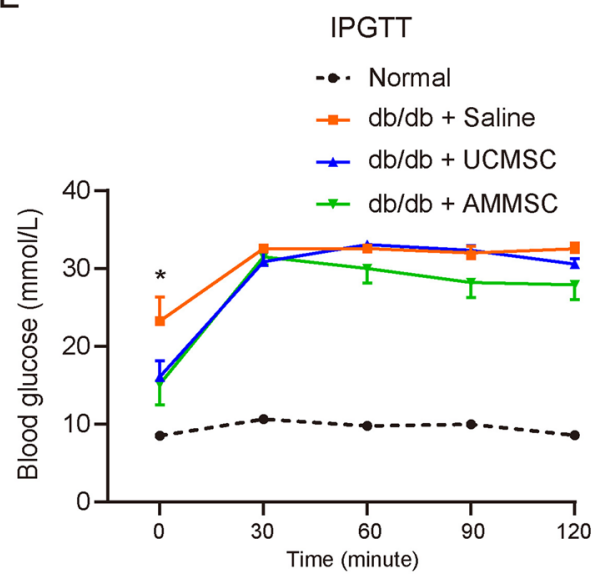

B

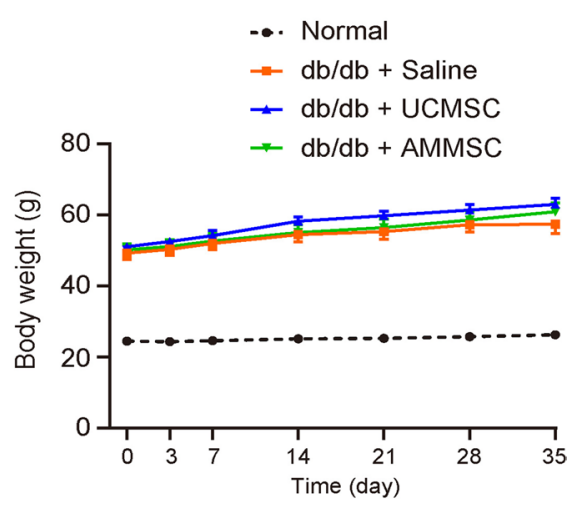

D

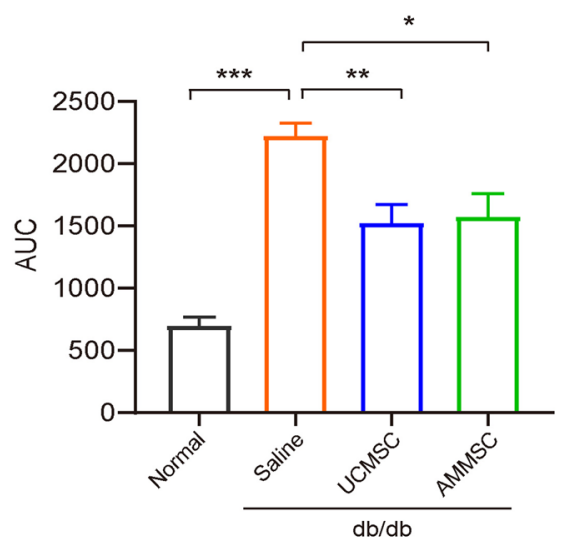

F

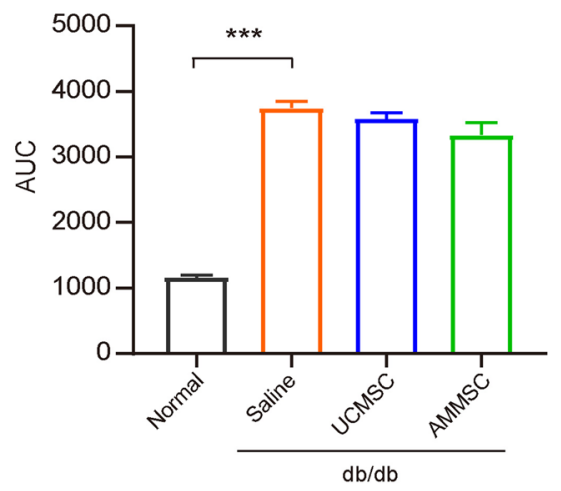




\section{MSCs Injection Decreased Lipid Deposition and Promoted Glycogen Synthesis in the Liver of $\mathrm{db} /$ db Mice}

Upon the pivotal roles of liver in regulating glycolipid metabolism, we then explored whether MSCs benefit liver functions in $\mathrm{db} / \mathrm{db}$ mice. Both AMMSCs and UCMSCs comparably reduced the ratio of liver to body weight in $\mathrm{db} / \mathrm{db}$ mice, when compared to the saline control (Fig. 4A). On the contrary, either AMMSC or UCMSC injection had no influence on the ratio of EWAT to body weight (Fig. 4B). Either AMMSC or UCMSC infusion
Fig. 4 MSC administration decreased lipid accumulation and enhanced glycogen storage in the liver of $\mathrm{db} / \mathrm{db}$ mice. The ratios of liver to body weight (A) and ratios of EWAT to body weight (B) were calculated in different group mice at five weeks after MSC or saline infusion. (C) Representative pictures of PAS staining in the liver sections of different group mice. Scale bar $=200 \mu \mathrm{m}$. (D) Quantification of PAS-positive areas in the livers of different group mice. (E) Representative pictures of $\mathrm{H} \& \mathrm{E}$ staining in mouse liver sections. Scale bar $=200 \mu \mathrm{m}$. (F) Quantification of lipid droplet areas in the livers of different group mice. Quantitative data are shown as mean \pm SEM $(n=5-7$ mice per group). $* \mathrm{P}<0.05, * * \mathrm{P}<0.01$, $* * * \mathrm{P}<0.001$
A

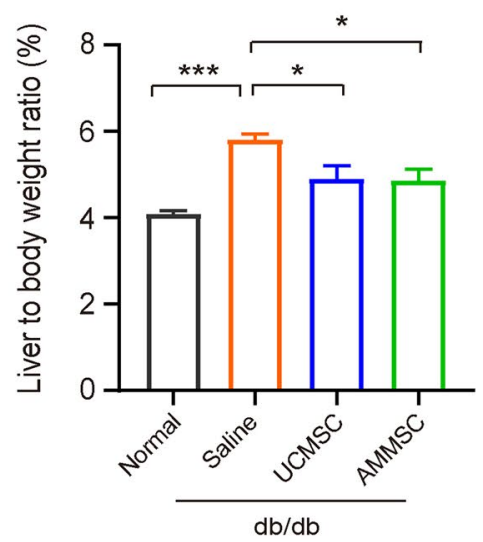

C

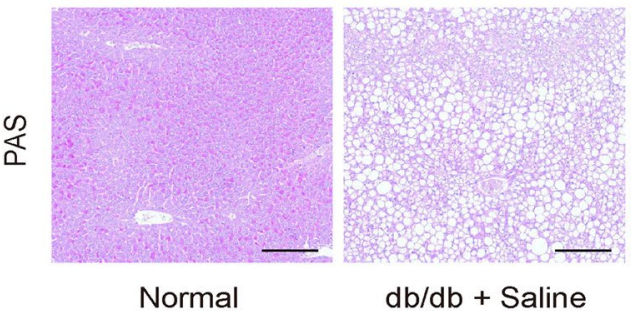

D

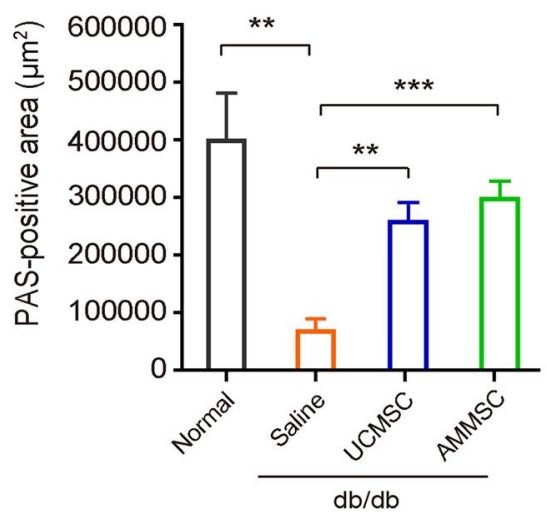

E

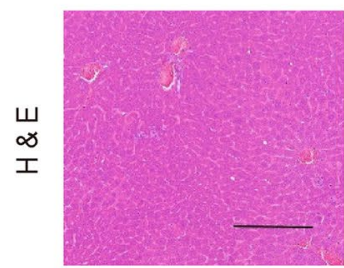

Normal
B
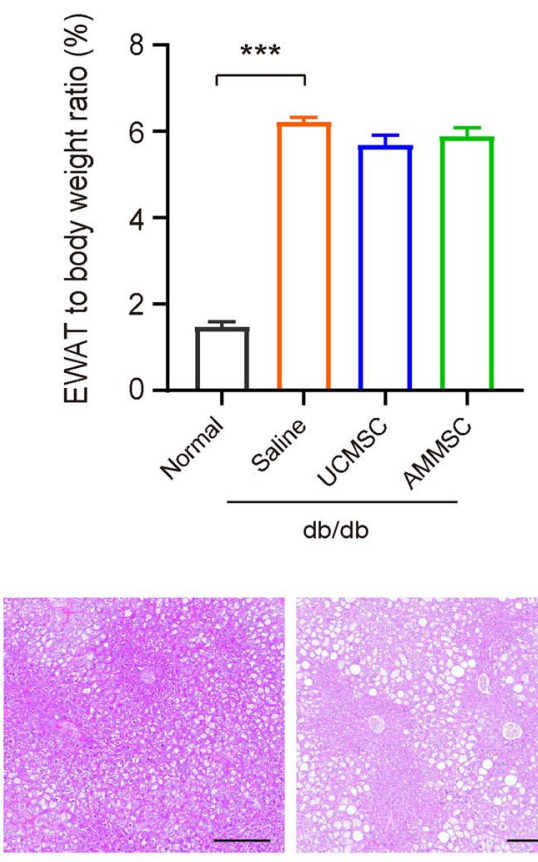

$d b / d b+U C M S C$

$d b / d b+A M M S C$

F

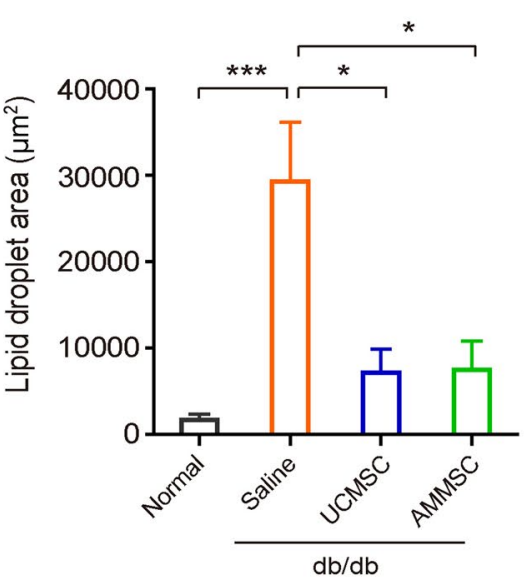

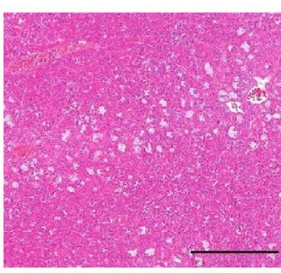

$d b / d b+U C M S C$

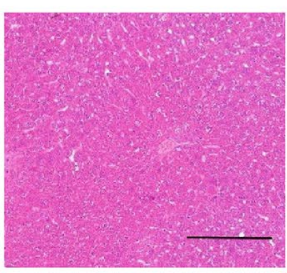

$\mathrm{db} / \mathrm{db}+\mathrm{AMMSC}$ 
could greatly restore the glycogen storage capacity of liver in $\mathrm{db} / \mathrm{db}$ mice (Fig. 4C and D). Meanwhile, in comparison to the saline control, both AMMSCs and UCMSCs dramatically reduced the number of hepatic lipid droplets in $\mathrm{db} / \mathrm{db}$ mice (Fig. 4E and F). These results implied that the infusion of the same donor-derived AMMSCs and UCMSCs generated comparable improvement on hepatic glycolipid metabolism in $\mathrm{db} / \mathrm{db}$ mice.

\section{MSC Infusion Enhanced Akt Phosphorylation and Improved Glycolipid Metabolic Dysfunction and Inflammation in the Liver of $\mathbf{d b} / \mathbf{d b}$ Mice}

Due to the hepatic protective effects observed in MSCsinfused $\mathrm{db} / \mathrm{db}$ mice, we specifically focused on the liver to explore cellular and molecular mechanisms underlying above anti-diabetic effects of AMMSCs and UCMSCs. In further support of the improved insulin sensitivity, insulin signaling was enhanced in the liver of MSCs-infused db/ $\mathrm{db}$ mice, as evidenced by the increased phosphorylation of Akt compared to saline-injected mice (Fig. 5A and B) (Supplementary Fig. 1). Consistent with increased hepatic glycogen storage and insulin sensitivity, hepatic mRNA expression level of glucose-6-phosphatase (G6PC), a key gluconeogenic gene, was significantly reduced in MSCsinjection $\mathrm{db} / \mathrm{db}$ mice when compared to saline controls (Fig. 5C). In agreement with the dramatic reduction of hepatic lipid accumulation in MSCs-treated $\mathrm{db} / \mathrm{db}$ mice (Fig. 4E and F), the mRNA expression levels of lipogenic genes fatty acid synthase (FASN) and sterol regulatory element-binding protein 1 (SREBP-1) were reduced in the liver of MSCs-infused db/db mice when compared to saline-injected mice (Fig. 5D and E). By contrast, the mRNA level of hepatic peroxisome proliferator-activated receptor $\alpha(P P A R \alpha)$, a key lipolytic gene, was significantly upregulated in MSCs-infused db/db mice (Fig. 5F).

Chronic low-grade inflammation in the liver contributes to hepatic glycolipid metabolic dysfunction and IR. Expectedly, the mRNA levels of macrophage marker gene $F 4 / 80$ and pro-inflammatory genes $I F N-\gamma$ and tumor necrosis factor- $\alpha(T N F-\alpha)$ were obviously increased in the liver of $\mathrm{db} / \mathrm{db}$ mice, when compared to normal mice (Fig. 5G-I). MSC administration could reverse the upregulated mRNA levels of hepatic $F 4 / 80$ and pro-inflammatory genes except $I F N-\gamma$ in the liver of AMMSCs-injected $\mathrm{db} / \mathrm{db}$ mice (Fig. 5G and H). Overall, these results suggested that both AMMSCs and UCMSCs shared similar hepatoprotective mechanisms via the enhancement of Akt phosphorylation, improvement of glycolipid metabolism, and reduction of inflammation in the liver of $\mathrm{db} / \mathrm{db}$ mice.

\section{Discussion}

It is essential to identify the ideal source of MSCs for maximizing their therapeutic efficacy in human diseases such as endocrine, neurodegenerative, and autoimmune disorders. Although produced under the uniform and strict good manufacturing practice processes, MSCs are a heterogeneous population of cells from biological properties to therapeutic efficiency, highly depending on donor source, tissue origin, and cultural condition [2, 39]. Despite originating from the same foetus, AMMSCs and UCMSCs could exhibit remarkable differences at diverse aspects including differentiation potential, paracrine factors and immunomodulatory capacity $[21-24,40]$. Human UCMSCs have been shown to proliferate faster than different donor-derived AMMSCs in serum-contained medium and chemically defined serum-free media [21-23]. Here, we observed that human UCMSCs did exhibit greater proliferative rate than AMMSCs isolated from the same donor. These data suggest that the higher proliferation capacity of human UCMSCs than AMMSCs may represent an intrinsic character. In line with the observed proliferative differences, we found that there is a higher $H G F$ mRNA level in UCMSCs compared to AMMSCs. HGF, a multifunctional cytokine, is enriched in the conditioned medium of human MSCs [41, 42], and is already reported to facilitate the proliferation of human BM- and arteriesderived MSCs in a concentration-dependent manner [43, 44]. Upon the promotion of HGF on angiogenesis [41], human UCMSCs might be more suitable for the treatment of diabetes-related vascular diseases such as diabetic nephropathy and retinopathy. IFN- $\gamma$, a key cytokine involved in adaptive and innate immune responses, can raise the immunosuppressive potency of human MSCs via preconditioning strategy. IFN- $\gamma$-preconditioned UCMSCs have been reported to exert better therapeutic efficiency in rodent animal models of several inflammatory disorders such as colitis and multiple sclerosis $[45,46]$. Consistent with previous observation [47], our results revealed that mRNA expression levels of $I L-6$ and $I D O I$ were significantly increased in IFN- $\gamma$-pretreated AMMSCs and UCMSCs, suggesting the reliability of IFN- $\gamma$-primed cell model applied in this study. Of note, in response to IFN$\gamma$, the same donor-derived AMMSCs and UCMSCs were found to display similar expression patterns of growth and anti-inflammatory factors except the $I G F 1$ with a greater expression level in AMMSCs. Because of the protective effects of IGF1 on MSC survival and functions upon exposure to oxidative and inflammatory stresses [48, 49], it is highly possible that AMMSCs may survival better than UCMSCs in IFN- $\gamma$-enriched inflammatory environments and thus exhibit a stronger therapeutic potential 
Fig. 5 MSC administration improved Akt phosphorylation and glycolipid metabolism dysfunction and reduced inflammation in the liver. (A) Western blotting analysis of Akt and p-Akt levels in the livers of different group mice. (B) Quantification of the ratio of p-Akt to Akt protein level in mouse livers. $n=4$ mice per group. (C-I) Gene expression patterns of $G 6 P C(\mathbf{C}), F A S N$ (D), SREBP-1 C (E), PPAR- $\alpha$ (F), $F 4 / 80(\mathbf{G}), I F N-\gamma(\mathbf{H})$ and $T N F-\alpha(\mathbf{I})$ in the livers of different group mice. $\mathrm{n}=6-7$ mice per group. Quantitative data are displayed as mean \pm SEM. $* P$ $<0.05, * * * P<0.001$
A

B

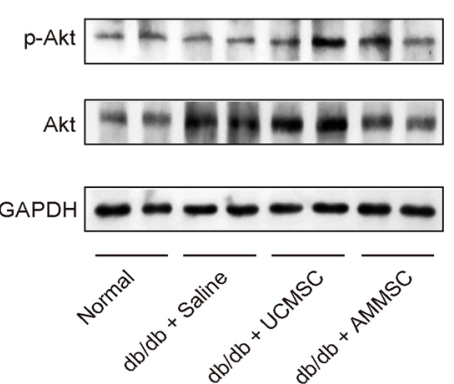

D

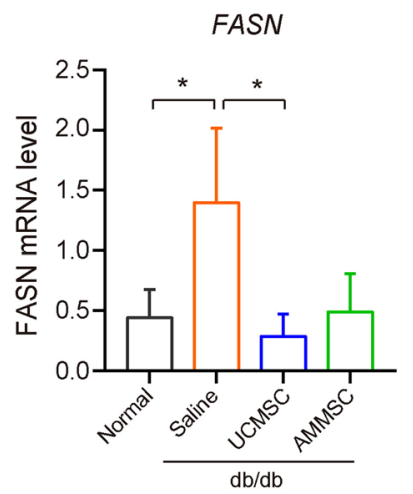

G

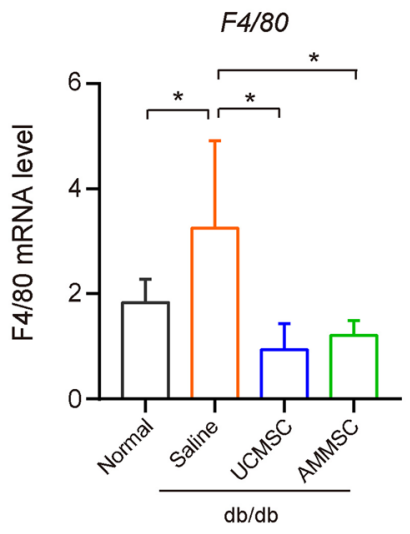

E

$\mathrm{H}$
C
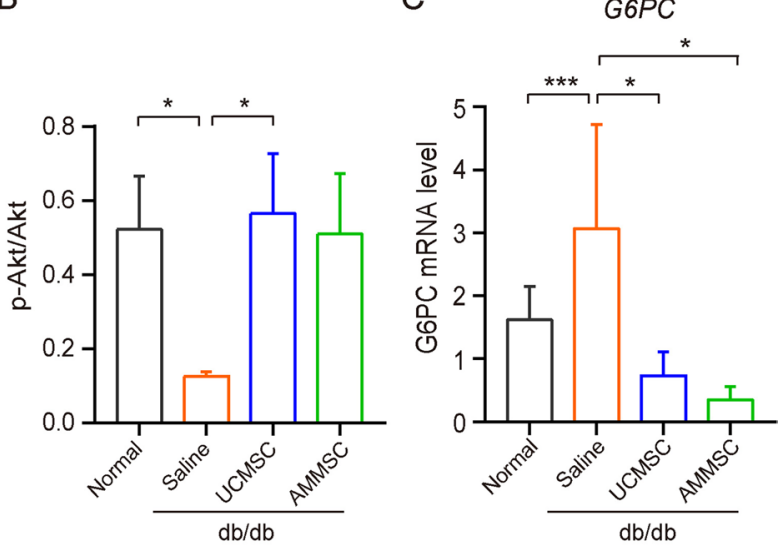

F
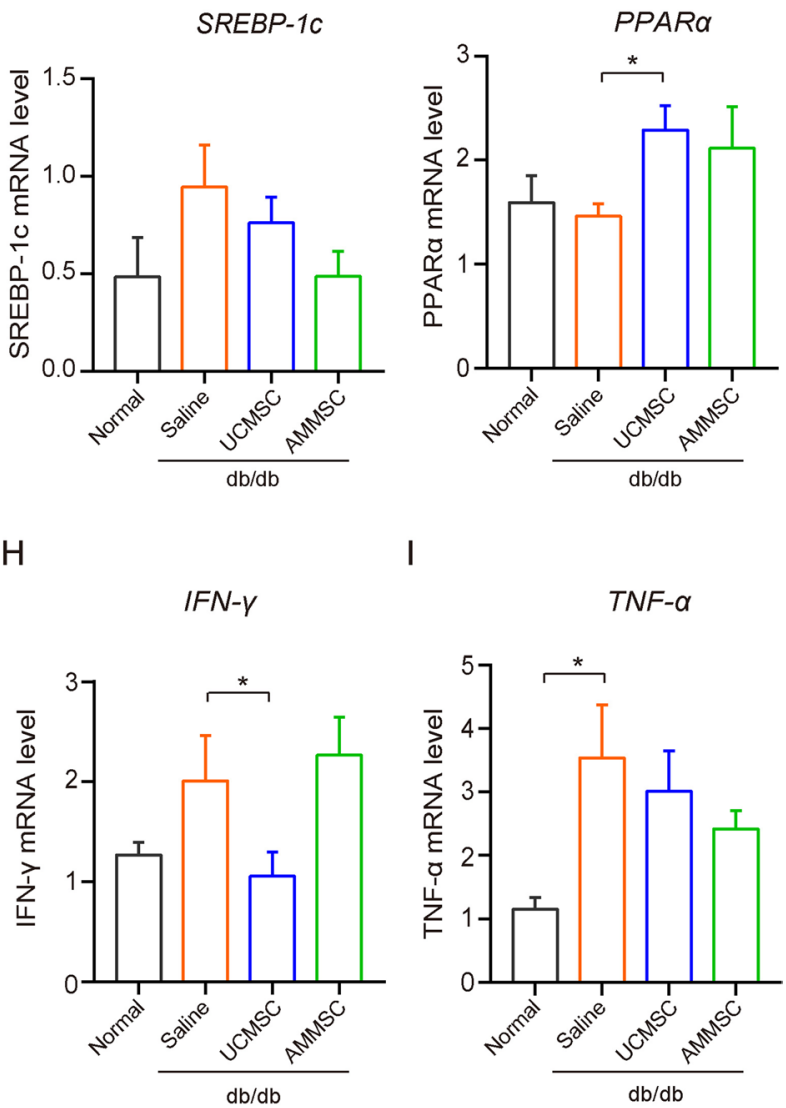

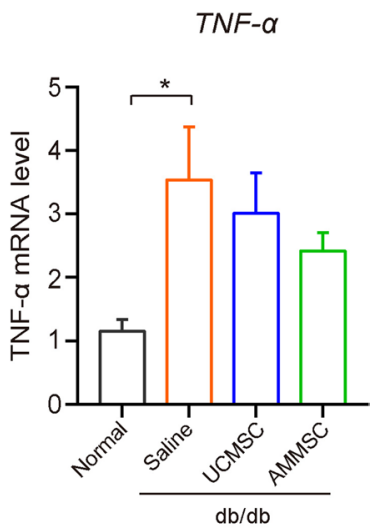

in IFN- $\boldsymbol{\gamma}$-dominated inflammatory diseases. To further understand the biological differences of AMMSCs and UCMSCs, more functional studies should be required to determine optimal MSC source for treating specific diseases. Besides indistinguishable differentiation potential into osteocytes, chondrocytes and adipocytes observed in this study, it would be necessary to evaluate the ability of AMMSCs and UCMSCs differentiating into other types of cells such as endothelial cells, cardiomyocytes, smooth muscle cells, hepatocytes, and insulin-producing cells.
Reduced liver inflammation contributes to the alleviation of AMMSCs and UCMSCs on T2DM symptoms in $\mathrm{db} /$ $\mathrm{db}$ mice. Chronic liver inflammation is a key contributor to the development of systemic IR and diabetic complications [28, 39]. Indeed, we observed higher mRNA levels of macrophage marker gene $F 4 / 80$ and pro-inflammatory genes $I F N-\gamma$ and $T N F-\alpha$ in the liver of $\mathrm{db} / \mathrm{db}$ mice when compared to the normal control, indicating a severe degree of hepatic inflammation in $\mathrm{db} / \mathrm{db}$ mice. Our data revealed that both AMMSC and UCMSC injection comparably decreased the 
expression level of hepatic $F 4 / 80 \mathrm{in} \mathrm{db} / \mathrm{db}$ mice, suggesting that the number of liver macrophages in $\mathrm{db} / \mathrm{db}$ mice declines after MSC application. Hepatic macrophages consist of two ontogenically distinct populations, namely circulating monocyte-derived macrophages and liver-specific macrophages (also called Kupffer cells) [50]. Because of the co-expression of F4/80 in Kupffer cells and monocytederived macrophages [51], further researches will be needed to investigate which subpopulation of hepatic macrophages could be affected by AMMSC and UCMSC infusion. Among T2DM-associated inflammatory factors, IFN- $\gamma$ is one of most important pro-inflammatory cytokines contributing to the pathogenesis of T2DM and systemic IR [52-54]. IFN$\gamma$, mostly generated by natural killer (NK) cells and CD4 ${ }^{+}$ $\mathrm{T}$ cells, participates in the pathogenesis of IR and T2DM through destroying skeletal muscle homeostasis and impairing lipid metabolism and insulin signal transduction in adipose cells [54]. In this study, we found that UCMSCs rather than AMMSCs could significantly inhibit the pathological up-regulation of hepatic $I F N-\gamma$ level in db/db mice, suggesting that these two kinds of MSCs may exhibit different regulatory patterns on the number of IFN- $\gamma$-generating immune cells. It will also be interesting to further explore which kind of immune cells mainly accounts for MSCs-induced IFN- $\gamma$ reduction by virtue of co-culture system of UCMSCs and particular immune cells.

AMMSCs and UCMSCs could comparably alleviate T2DM symptoms by largely restoring dysfunctional glycolipid metabolism in the liver of $\mathrm{db} / \mathrm{db}$ mice. Liver critically affects blood glucose homeostasis via the regulation of glucose uptake, degradation, synthesis and storage [6]. Thus, liver metabolic dysfunctions including low glucose utilization and excessive fat accumulation are believed as the major contributor to hyperglycemia and systemic IR in diabetes. Hepatic IR, whose occurrence precedes systemic IR, is an important factor triggering the abnormality of hepatic glycolipid metabolism in T2DM individuals [7]. Due to IR and low glucose uptake capacity, the hepatocytes of T2DM individuals turn to employ fat metabolism for their energy and nutrient requirement through intensifying de novo lipogenesis $[7,30]$, consequently conducing to excess lipid accumulation in liver cells. Indeed, we found that there were significantly increased liver to body weight ratio and hepatic lipid accumulation but greatly reduced hepatic glycogen deposit in the $\mathrm{T} 2 \mathrm{DM} \mathrm{db} / \mathrm{db}$ mice when compared to the normal control. However, either AMMSC or UCMSC administration could significantly reverse the dysfunction of glycolipid metabolism observed in $\mathrm{db} / \mathrm{db}$ mice, thereby leading to the improvement of hyperglycemia and systemic insulin sensitivity. Mechanistically, we found that both AMMSC and UCMSC infusion reversed abnormal up-regulation of lipogenesis-correlated genes $F A S N$ and SREBP-1 in the liver of $\mathrm{db} / \mathrm{db}$ mice, implying that T2DM-related hyperactivation of de novo lipogenesis is restrained. On the contrary, either AMMSC or UCMSC injection could significantly increase the mRNA level of hepatic PPAR- $\alpha$ that promotes fatty acid $\beta$-oxidation, suggesting that PPAR- $\alpha$-involved lipid degradation may also conduce to the reduction of liver fat content in MSCs-infused $\mathrm{db} / \mathrm{db}$ mice. Accordingly, AMMSCs and UCMSCs may act as very promising therapeutic agents to treat metabolic dysfunction-associated fatty liver diseases. Apart from the hyperactive lipogenesis, liver cells of diabetic individuals are described to compensatorily reinforce de novo glucose production via G6PC-involved gluconeogenesis [6]. Indeed, we found a greater $G 6 P C$ mRNA level in the liver of $d b / d b$ mice when compared to the normal mice. However, this abnormally high level of hepatic G6PC was reversed by single-dose infusion of either AMMSCs or UCMSCs, which may arise from improved hepatic IR and glucose uptake in MSCs-injected db/db mice. In favor of this speculation, our data showed that both AMMSCs and UCMSCs could increase Akt phosphorylation, a key insulin sensitivity index, in the liver of $\mathrm{db} / \mathrm{db}$ mice. Nevertheless, the precise mechanisms underlying the alleviation of MSC infusion on T2DM syndromes remain elusive. Growing reports showed that MSCs-derived exosomes containing numerous bioactive molecules, rather than cell-cell contact, predominantly mediate the therapeutic outcomes of MSCs in T2DM rodent models [2, 5, 55]. Human UCMSCs-released IL-6 has been manifested to mediate the alleviation of UCMSCs on hyperglycemia and IR in T2DM rats through enhancing macrophage polarization in the liver and adipose tissues [56]. Consistent with this report, the intraperitoneal application of recombinant human IL-6 obviously decreases plasma triglyceride and cholesterol concentrations, increases hepatic PPAR- $\alpha$ level, and reduces hepatic SREBP-1 expression in C57BL/6 mice [57]. Therefore, it is reasonable to infer that MSCs-secreted IL- 6 contributes largely to the amelioration of AMMSCs and UCMSCs on T2DM symptoms and liver metabolic dysfunctions in $\mathrm{db} / \mathrm{db}$ mice. However, further experiments are needed to verify this hypothesis via the injection of IL-6-knockout AMMSCs or UCMSCs in T2DM animal models.

Besides tissue sources, the safety and therapeutic efficiency of MSCs is also greatly dependent on cell dosage, injection frequency, and the interval between injections [1, $39,58]$, which needs extensive exploration to determine the optimal scheme for clinical application. In general, multiple injections of MSCs give rise to more effective and durable therapeutic effects in the treatment of human diseases. Thus, apart from single-dose injection of AMMSCs or UCMSCs examined in our study, it is of great importance to further assess the safety and efficacy of different dosage and/or multiple injections of AMMSCs and UCMSCs on the alleviation of T2DM syndromes. In this regard, we preliminarily tested intravenous infusion of high-dose AMMSCs or UCMSCs 
Fig. 6 Working model of perinatal MSCs in alleviating T2DM symptoms. Single-dose intravenous infusion of either AMMSCs or UCMSCs from the same donor comparably alleviates T2DM symptoms in $\mathrm{db} / \mathrm{db}$ mice through improving glycolipid metabolism, enhancing insulin sensitivity, and decreasing inflammation in the liver

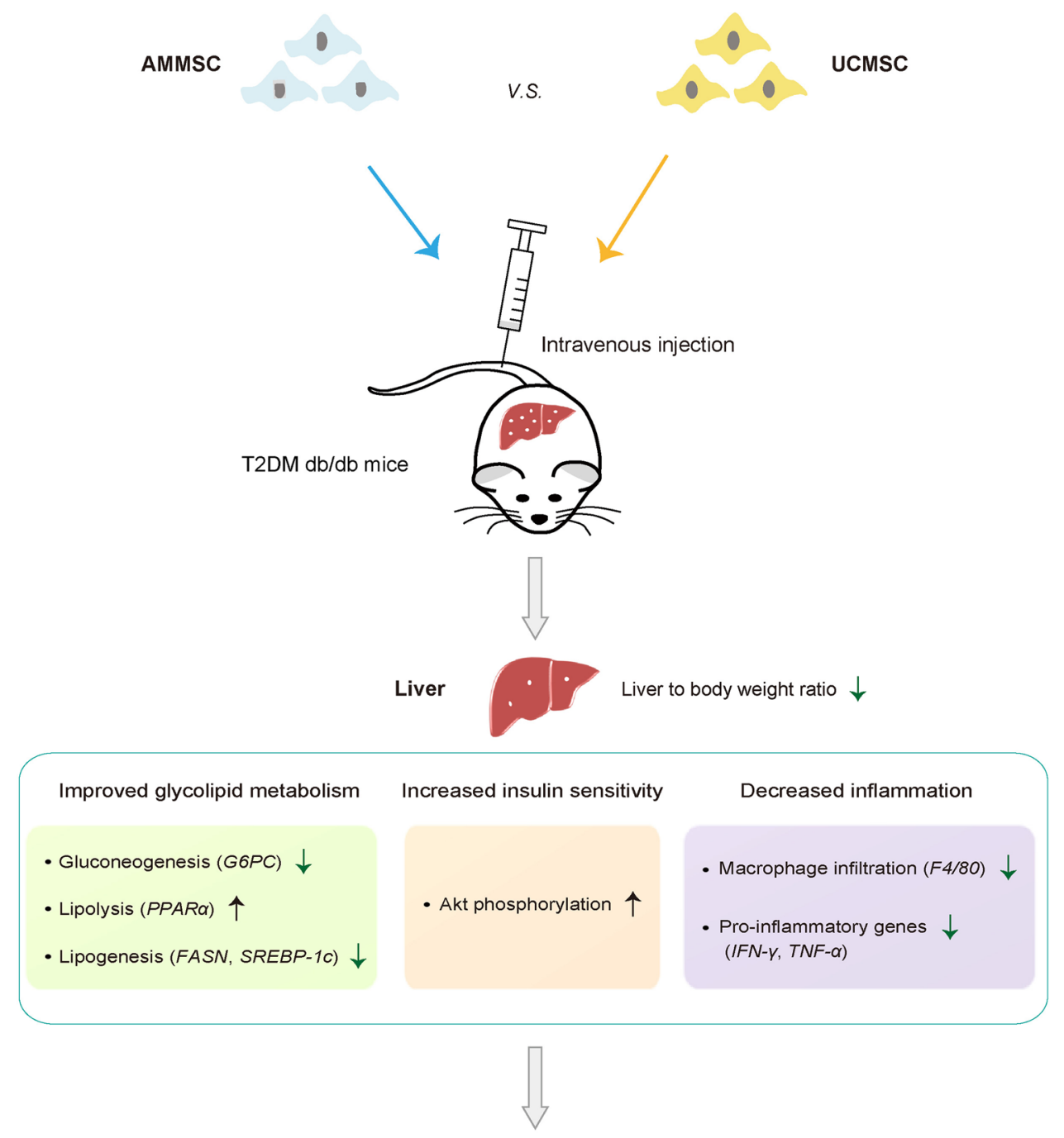

Amelioration of T2DM symptoms $\left(5 \times 10^{6}\right.$ cells/mouse), which caused high mortality of $\mathrm{db} / \mathrm{db}$ mice due to acute pulmonary embolism (data not shown), implying that other sub-high dosages (e.g., $3 \times 10^{6}$ cells/ mouse) need to be determined later. Moreover, in view of clinical application of AMMSCs and UCMSCs for T2DM treatment, it's necessary to further investigate in vivo biodistribution, retention time, and differentiation ability toward insulin-producing cells of these human MSCs in the T2DM animal model.

\section{Conclusions}

In summary, we found that single-dose intravenous administration of the same donor-derived AMMSCs and UCMSCs have a similar ameliorating effect on severe T2DM symptoms including hyperglycemia and systemic IR in $\mathrm{db} / \mathrm{db}$ mice. These comparable anti-diabetic benefits of AMMSCs and UCMSCs could be ascribed to the improved glycolipid metabolism, increased insulin sensitivity, and decreased inflammation in the liver of $\mathrm{db} / \mathrm{db}$ mice (Fig. 6).

Supplementary Information The online version contains supplementary material available at https://doi.org/10.1007/s12015-021-10320-w.

Author Contribution Z.F. W., J.M. F., X.Y. W., S.H. D., W. C., Y.H. G., and Z. L. conducted the experiments; Z.F. W. and H.S. L. wrote the manuscript with help from all the authors. Z.F. W. and H. Z. designed, supervised, and coordinated all aspects of this project. All authors approved the manuscript prior to submission and publication.

Funding This study was supported by grants from industry-academiaresearch of SNC and Tongji University (Grant No. SNC2018007).

Data Availability The data that support the findings of this study are available from the corresponding author upon reasonable request.

Code Availability Not applicable. 


\section{Declarations}

Conflict of Interest The authors declare that there are no conflicts of interest.

Ethics Approval The care and use of human AMMSCs and UCMSCs were approved by the Tenth People's Hospital of Tongji University. The donor has signed the informed consent.

All animal experiments were reviewed and approved by the Laboratory Animal Care and Use Committee of Tongji University.

Consent to Participate Informed consent was obtained from all individual participants included in the study.

Consent for Publication All participants have approved this article to be published.

\section{References}

1. Kamal, M. M., \& Kassem, D. H. (2020). Therapeutic potential of Wharton's Jelly Mesenchymal Stem Cells for Diabetes: achievements and challenges. Frontiers in Cell and Developmental Biology, 8,16

2. Li, H., Zhu, H., Ge, T., Wang, Z., \& Zhang, C. (2021). Mesenchymal stem cell-based therapy for diabetes mellitus: enhancement strategies and future perspectives. Stem Cell Reviews and Reports, 17(5), 1552-1569

3. Keane, K. N., Calton, E. K., Carlessi, R., Hart, P. H., \& Newsholme, P. (2017). The bioenergetics of inflammation: insights into obesity and type 2 diabetes. European Journal of Clinical Nutrition, 71(7), 904-912

4. Qi, Y., Ma, J., Li, S., \& Liu, W. (2019). Applicability of adiposederived mesenchymal stem cells in treatment of patients with type 2 diabetes. Stem Cell Research \& Therapy, 10(1), 274

5. He, Q., Wang, L., Zhao, R., Yan, F., Sha, S., Cui, C., et al. (2020). Mesenchymal stem cell-derived exosomes exert ameliorative effects in type 2 diabetes by improving hepatic glucose and lipid metabolism via enhancing autophagy. Stem Cell Research \& Therapy, 11(1), 223

6. Jiang, S., Young, J. L., Wang, K., Qian, Y., \& Cai, L. (2020). Diabetic-induced alterations in hepatic glucose and lipid metabolism: The role of type 1 and type 2 diabetes mellitus (Review). Molecular Medicine Reports, 22(2), 603-611

7. Perry, R. J., Samuel, V. T., Petersen, K. F., \& Shulman, G. I. (2014). The role of hepatic lipids in hepatic insulin resistance and type 2 diabetes. Nature, 510(7503), 84-91

8. Zhang, Y., Gao, S., Liang, K., Wu, Z., Yan, X., Liu, W., et al. (2021). Exendin-4 gene modification and microscaffold encapsulation promote self-persistence and antidiabetic activity of MSCs. Science Advances, 7(27), eabi4379

9. Chellappan, D. K., Yap, W. S., Bt Ahmad Suhaimi, N. A., Gupta, G., \& Dua, K. (2018). Current therapies and targets for type 2 diabetes mellitus. Panminerva Medica, 60(3), 117-131

10. Chaudhury, A., Duvoor, C., Reddy Dendi, V. S., Kraleti, S., Chada, A., Ravilla, R., et al. (2017). Clinical review of antidiabetic drugs: implications for Type 2 Diabetes Mellitus Management. Frontiers in Endocrinology, 8, 6

11. Pittenger, M. F., Discher, D. E., Péault, B. M., Phinney, D. G., Hare, J. M., \& Caplan, A. I. (2019). Mesenchymal stem cell perspective: cell biology to clinical progress. NPJ Regenerative Medicine, 4,22
12. Brown, C., McKee, C., Bakshi, S., Walker, K., Hakman, E., Halassy, S., et al. (2019). Mesenchymal stem cells: Cell therapy and regeneration potential. Journal of Tissue Engineering and Regenerative Medicine, 13(9), 1738-1755

13. Osipova, O., Saaya, S., Karpenko, A., Zakian, S., \& Aboian, E. (2019). Cell therapy of critical limb ischemia-problems and prospects. Vasa, 48(6), 461-471

14. Wang, L. T., Ting, C. H., Yen, M. L., Liu, K. J., Sytwu, H. K., Wu, K. K., et al. (2016). Human mesenchymal stem cells (MSCs) for treatment towards immune- and inflammation-mediated diseases: review of current clinical trials. Journal of Biomedical Science, 23(1), 76

15. Qadura, M., Terenzi, D. C., Verma, S., Al-Omran, M., \& Hess, D. A. (2018). Concise review: cell therapy for critical limb ischemia: an integrated review of preclinical and clinical studies. Stem Cells, $36(2), 161-171$

16. Kim, J., Lee, Y., Lee, S., Kim, K., Song, M., \& Lee, J. (2020). Mesenchymal stem cell therapy and Alzheimer's Disease: current status and future perspectives. Journal of Alzheimer's Disease, $77(1), 1-14$

17. Qu, W., Wang, Z., Hare, J. M., Bu, G., Mallea, J. M., Pascual, J. M., et al. (2020). Cell-based therapy to reduce mortality from COVID-19: Systematic review and meta-analysis of human studies on acute respiratory distress syndrome. Stem Cells Translational Medicine, 9(9), 1007-1022

18. Xiong, J., Chen, L., Zhang, L., Bao, L., \& Shi, Y. (2021). Mesenchymal stromal cell-based therapy: a promising approach for severe COVID-19. Cell Transplantation, 30, 963689721995455

19. Torre, P., \& Flores, A. I. (2020). Current status and future prospects of perinatal stem cells. Genes (Basel), 12(1), 6

20. Shen, C., Yang, C., Xu, S., \& Zhao, H. (2019). Comparison of osteogenic differentiation capacity in mesenchymal stem cells derived from human amniotic membrane (AM), umbilical cord (UC), chorionic membrane (CM), and decidua (DC). Cell \& Bioscience, 9,17

21. Ma, J., Wu, J., Han, L., Jiang, X., Yan, L., Hao, J., et al. (2019). Comparative analysis of mesenchymal stem cells derived from amniotic membrane, umbilical cord, and chorionic plate under serum-free condition. Stem Cell Research \& Therapy, 10(1), 19

22. Araújo, A. B., Salton, G. D., Furlan, J. M., Schneider, N., Angeli, M. H., Laureano, Á., et al. (2017). Comparison of human mesenchymal stromal cells from four neonatal tissues: Amniotic membrane, chorionic membrane, placental decidua and umbilical cord. Cytotherapy, 19(5), 577-585

23. Guan, Y. T., Xie, Y., Li, D. S., Zhu, Y. Y., Zhang, X. L., Feng, Y. L., et al. (2019). Comparison of biological characteristics of mesenchymal stem cells derived from the human umbilical cord and decidua parietalis. Molecular Medicine Reports, 20(1), 633-639

24. Dabrowski, F. A., Burdzinska, A., Kulesza, A., Sladowska, A., Zolocinska, A., Gala, K., et al. (2017). Comparison of the paracrine activity of mesenchymal stem cells derived from human umbilical cord, amniotic membrane and adipose tissue. Journal of Obstetrics and Gynaecology Research, 43(11), 1758-1768

25. Mu, Y., Wu, X., \& Hao, Z. (2018). Comparative evaluation of mesenchymal stromal cells from umbilical cord and amniotic membrane in xeno-free conditions. BMC Molecular and Cell Biology, 19(1), 27

26. El-Badawy, A., \& El-Badri, N. (2016). Clinical efficacy of stem cell therapy for Diabetes Mellitus: a meta-analysis. PLoS One, 11(4), e0151938

27. Si, Y., Zhao, Y., Hao, H., Liu, J., Guo, Y., Mu, Y., et al. (2012). Infusion of mesenchymal stem cells ameliorates hyperglycemia in type 2 diabetic rats: identification of a novel role in improving insulin sensitivity. Diabetes, 61(6), 1616-1625

28. Sun, X., Hao, H., Han, Q., Song, X., Liu, J., Dong, L., et al. (2017). Human umbilical cord-derived mesenchymal stem cells ameliorate 
insulin resistance by suppressing NLRP3 inflammasome-mediated inflammation in type 2 diabetes rats. Stem Cell Research \& Therapy, $8(1), 241$

29. Yin, Y., Hao, H., Cheng, Y., Gao, J., Liu, J., Xie, Z., et al. (2018). The homing of human umbilical cord-derived mesenchymal stem cells and the subsequent modulation of macrophage polarization in type 2 diabetic mice. International Immunopharmacology, 60, 235-245

30. Li, B., Cheng, Y., Yu, S., Zang, L., Yin, Y., Liu, J., et al. (2019). Human umbilical cord-derived mesenchymal stem cell therapy ameliorates nonalcoholic fatty liver disease in obese Type 2 Diabetic mice. Stem Cells International, 2019, 8628027

31. Kim, S. W., Zhang, H. Z., Guo, L., Kim, J. M., \& Kim, M. H. (2012). Amniotic mesenchymal stem cells enhance wound healing in diabetic NOD/SCID mice through high angiogenic and engraftment capabilities. PLoS One, 7(7), e41105

32. Kim, J., Park, S., Kang, H. M., Ahn, C. W., Kwon, H. C., Song, J. H., et al. (2012). Human insulin secreted from insulinogenic xenograft restores normoglycemia in type 1 diabetic mice without immunosuppression. Cell Transplantation, 21(10), 2131-2147

33. Kadam, S. S., Sudhakar, M., Nair, P. D., \& Bhonde, R. R. (2010). Reversal of experimental diabetes in mice by transplantation of neo-islets generated from human amnion-derived mesenchymal stromal cells using immuno-isolatory macrocapsules. Cytotherapy, 12(8), 982-991

34. Chisari, G., Chisari, E. M., Borzi, A. M., Grasso, A., \& Chisari, C. G. (2017). Amniotic membrane use in type 2 diabetes patients with chronic ulcers: Microbiological evaluation and therapeutic benefits. Acta Medica Mediterranea, 33(3), 431-435

35. Araújo, A. B., Furlan, J. M., Salton, G. D., Schmalfuss, T., Röhsig, L. M., Silla, L. M. R., et al. (2018). Isolation of human mesenchymal stem cells from amnion, chorion, placental decidua and umbilical cord: comparison of four enzymatic protocols. Biotechnology Letters, 40(6), 989-998

36. Ryan, J. M., Barry, F., Murphy, J. M., \& Mahon, B. P. (2007). Interferon-gamma does not break, but promotes the immunosuppressive capacity of adult human mesenchymal stem cells. Clinical \& Experimental Immunology, 149(2), 353-363

37. Boland, L., Burand, A. J., Brown, A. J., Boyt, D., Lira, V. A., \& Ankrum, J. A. (2018). IFN- $\gamma$ and TNF- $\alpha$ pre-licensing protects mesenchymal stromal cells from the pro-inflammatory effects of palmitate. Molecular Therapy, 26(3), 860-873

38. Klinker, M. W., Marklein, R. A., Lo Surdo, J. L., Wei, C. H., \& Bauer, S. R. (2017). Morphological features of IFN- $\gamma$-stimulated mesenchymal stromal cells predict overall immunosuppressive capacity. Proceedings of the National Academy of Sciences of the United States of America, 114(13), E2598-E2607

39. Chen, J., Zheng, C. X., Jin, Y., \& Hu, C. H. (2021). Mesenchymal stromal cell-mediated immune regulation: A promising remedy in the therapy of type 2 diabetes mellitus. Stem Cells, 39(7), 838-852

40. Hendrijantini, N., \& Hartono, P. (2019). Phenotype characteristics and osteogenic differentiation potential of Human Mesenchymal Stem Cells Derived from Amnion Membrane (HAMSCs) and Umbilical Cord (HUC-MSCs). Acta Informatica Medica, 27(2), 72-77

41. Lu, H., Wang, F., Mei, H., Wang, S., \& Cheng, L. (2018). Human adipose mesenchymal stem cells show more efficient angiogenesis promotion on endothelial colony-forming cells than umbilical cord and endometrium. Stem Cells International, 2018, 7537589

42. Jia, Y., Cao, N., Zhai, J., Zeng, Q., Zheng, P., Su, R., et al. (2020). HGF mediates clinical-grade human umbilical cord-derived mesenchymal stem cells improved functional recovery in a senescenceaccelerated mouse model of Alzheimer's Disease. Advanced Science, 7(17), 1903809

43. Valente, S., Ciavarella, C., Pasanisi, E., Ricci, F., Stella, A., \& Pasquinelli, G. (2016). Hepatocyte growth factor effects on mesenchymal stem cells derived from human arteries: a novel strategy to accelerate vascular ulcer wound healing. Stem Cells International, 2016, 3232859

44. Cao, Z., Xie, Y., Yu, L., Li, Y., \& Wang, Y. (2020). Hepatocyte growth factor (HGF) and stem cell factor (SCF) maintained the stemness of human bone marrow mesenchymal stem cells (hBMSCs) during long-term expansion by preserving mitochondrial function via the PI3K/AKT, ERK1/2, and STAT3 signaling pathways. Stem Cell Research \& Therapy, 11(1), 329

45. Yu, Y., Yoo, S. M., Park, H. H., Baek, S. Y., Kim, Y. J., Lee, S., et al. (2019). Preconditioning with interleukin-1 beta and interferon-gamma enhances the efficacy of human umbilical cord blood-derived mesenchymal stem cells-based therapy via enhancing prostaglandin E2 secretion and indoleamine 2,3-dioxygenase activity in dextran sulfate sodium-induced colitis. Journal of Tissue Engineering and Regenerative Medicine, 13(10), 1792-1804

46. Zhou, X., Liu, X., Liu, L., Han, C., Xie, Z., Liu, X., et al. (2020). Transplantation of IFN- $\gamma$ primed hUCMSCs significantly improved outcomes of experimental autoimmune encephalomyelitis in a mouse model. Neurochemical Research, 45(7), 1510-1517

47. Kim, D. S., Jang, I. K., Lee, M. W., Ko, Y. J., Lee, D. H., Lee, J. W., et al. (2018). Enhanced immunosuppressive properties of human mesenchymal stem cells primed by interferon- $\gamma$. EBioMedicine, 28, 261-273

48. Liu, P., Feng, Y., Dong, D., Liu, X., Chen, Y., Wang, Y., et al. (2016). Enhanced renoprotective effect of IGF-1 modified human umbilical cord-derived mesenchymal stem cells on gentamicin-induced acute kidney injury. Scientific Reports, 6, 20287

49. Allahdadi, K. J., de Santana, T. A., Santos, G. C., Azevedo, C. M., Mota, R. A., Nonaka, C. K., et al. (2019). IGF-1 overexpression improves mesenchymal stem cell survival and promotes neurological recovery after spinal cord injury. Stem cell Research \& Therapy, 10(1), 146

50. Ju, C., \& Tacke, F. (2016). Hepatic macrophages in homeostasis and liver diseases: from pathogenesis to novel therapeutic strategies. Cellular \& Molecular Immunology, 13(3), 316-327

51. van den Berg, T. K., \& Kraal, G. (2005). A function for the macrophage F4/80 molecule in tolerance induction. Trends in Immunology, 26(10), 506-509

52. Akash, M. S. H., Rehman, K., \& Liaqat, A. (2018). Tumor necrosis factor-Alpha: role in development of insulin resistance and pathogenesis of Type 2 Diabetes Mellitus. Journal of Cellular Biochemistry, 119(1), 105-110

53. Schoenborn, J. R., \& Wilson, C. B. (2007). Regulation of interferongamma during innate and adaptive immune responses. Advances in Immunology, 96, 41-101

54. Li, P., Zhao, Y., Wu, X., Xia, M., Fang, M., Iwasaki, Y., et al. (2012). Interferon gamma (IFN- $\gamma$ ) disrupts energy expenditure and metabolic homeostasis by suppressing SIRT1 transcription. Nucleic Acids Research, 40(4), 1609-1620

55. Sun, Y., Shi, H., Yin, S., Ji, C., Zhang, X., Zhang, B., et al. (2018). Human mesenchymal stem cell derived exosomes alleviate Type 2 Diabetes Mellitus by reversing peripheral insulin resistance and relieving $\beta$-cell destruction. ACS Nano, 12(8), 7613-7628

56. Xie, Z., Hao, H., Tong, C., Cheng, Y., Liu, J., Pang, Y., et al. (2016). Human umbilical cord-derived mesenchymal stem cells elicit macrophages into an anti-inflammatory phenotype to alleviate insulin resistance in type 2 diabetic rats. Stem Cells, 34(3), 627-639

57. Hashizume, M., \& Mihara, M. (2011). IL-6 and lipid metabolism. Inflammation and Regeneration, 31(3), 325-333

58. Zang, L., Hao, H., Liu, J., Li, Y., Han, W., \& Mu, Y. (2017). Mesenchymal stem cell therapy in type 2 diabetes mellitus. Diabetology \& Metabolic Syndrome, 9, 36

Publisher's Note Springer Nature remains neutral with regard to jurisdictional claims in published maps and institutional affiliations. 